University of Nebraska - Lincoln

DigitalCommons@University of Nebraska - Lincoln

December 2001

\title{
Dilution-induced enhancement of the blocking temperature in exchange-bias heterosystems
}

\author{
Xi Chen \\ Laboratorium für Angewandte Physik, Gerhard-Mercator-Universita"t Duisburg, 47048 Duisburg, Germany \\ Christian Binek \\ University of Nebraska-Lincoln, cbinek@unl.edu
}

A. Hochstrat

Laboratorium fu"r Angewandte Physik, Gerhard-Mercator-Universita"t Duisburg, 47048 Duisburg, Germany

Wolfgang Kleemann

Laboratorium fu"r Angewandte Physik, Gerhard-Mercator-Universita"t Duisburg, 47048 Duisburg,

Germany, kleemann@uni-duisburg.de

Follow this and additional works at: https://digitalcommons.unl.edu/physicsbinek

Part of the Physics Commons

Chen, Xi; Binek, Christian; Hochstrat, A.; and Kleemann, Wolfgang, "Dilution-induced enhancement of the blocking temperature in exchange-bias heterosystems" (2001). Christian Binek Publications. 18.

https://digitalcommons.unl.edu/physicsbinek/18

This Article is brought to you for free and open access by the Research Papers in Physics and Astronomy at DigitalCommons@University of Nebraska - Lincoln. It has been accepted for inclusion in Christian Binek Publications by an authorized administrator of DigitalCommons@University of Nebraska - Lincoln. 


\title{
Dilution-induced enhancement of the blocking temperature in exchange-bias heterosystems
}

\author{
Xi Chen, Ch. Binek, A. Hochstrat, and W. Kleemann \\ Laboratorium für Angewandte Physik, Gerhard-Mercator-Universität Duisburg, 47048 Duisburg, Germany
}

(Received 28 August 2001; published 13 December 2001)

\begin{abstract}
The temperature dependence of the exchange bias field is investigated by superconducting quantum interference device magnetometry in $\mathrm{Fe}_{1-x} \mathrm{Zn}_{x} \mathrm{~F}_{2}(110) / \mathrm{Fe} 14 \mathrm{~nm} / \mathrm{Ag} 35 \mathrm{~nm}, x=0.4$. Its blocking temperature exhibits a significant enhancement with respect to the global ordering temperature $T_{N}=46.9 \mathrm{~K}$, of the bulk antiferromagnet $\mathrm{Fe}_{0.6} \mathrm{Zn}_{0.4} \mathrm{~F}_{2}$. The enhancement is attributed to fluctuations of the diamagnetic dilution which creates clusters on all length scales having a $\mathrm{Zn}$ dilution of $0 \leqslant x \leqslant 1$. While the infinite clusters give rise to the well-known Griffiths phase, finite clusters also provoke a local enhancement of the exchange bias. The temperature dependence of the integral exchange bias effect is modeled by averaging all local contributions of the antiferromagnetic surface magnetization which exhibit a surface critical behavior.
\end{abstract}

DOI: $10.1103 /$ PhysRevB.65.012415

Recently, the exchange bias (EB) effect has attracted a great deal of interest in basic research and materials science, ${ }^{1,2}$ owing to its relevance for technological applications. The EB effect describes a shift of the ferromagnetic hysteresis loop along the magnetic-field axis away from its origin at $H=0$, which is caused by the exchange coupling at the interface between ferromagnetic (FM) and antiferromagnetic (AF) materials after cooling or growth of the sample in a magnetic field to below the Néel temperature of the antiferromagnet.

Meiklejohn and Bean proposed a simple model of the EB effect which provides the basic, but powerful formula $H_{\mathrm{EB}}$ $=-J_{\mathrm{EB}} S_{\mathrm{AF}} S_{\mathrm{FM}} /\left(M_{\mathrm{FM}} t_{\mathrm{FM}}\right)$ for the exchange bias field. ${ }^{3,4}$ Here $S_{\mathrm{AF}}$ and $S_{\mathrm{FM}}$ are the AF and FM spins at the interface, $M_{\mathrm{FM}}$ and $t_{\mathrm{FM}}$ are the magnetization and the thickness of the FM layer, while $J_{\mathrm{EB}}$ is the interface coupling constant. The above formula suggests that the exchange bias effect vanishes at the Néel temperature $T_{N}$ of the bulk antiferromagnet. Note that, in general, the Curie temperature of the ferromagnet is much higher than $T_{N}$, and has no impact on the temperature dependence of $H_{\mathrm{EB}}$. A rare exception was reported in Refs. 5 and 6, where FM/AF bilayers with comparable critical temperatures $T_{c}$ and $T_{N}$ exhibit an enhanced exchange bias field on approaching $T_{c} \gtrsim T_{N}$. Typically, the blocking temperature $T_{B}$, i.e., the temperature of the vanishing exchange bias effect, is smaller than $T_{N} \cdot{ }^{1}$ This behavior is usually attributed to finite-size effects which are known, e.g., from the dependence of the EB effect on the AF layer thickness. ${ }^{4,7,8}$ Moreover Zaag et al. ${ }^{9}$ pointed out that $T_{B}$ does not track the ordering temperature in a simple manner. The authors of Ref. 10 showed that in $\mathrm{FeF}_{2}$-Fe bilayers near $T_{N}$ the temperature dependence of the exchange bias field follows the temperature dependence of the AF interface magnetic moment, which exhibits a power-law behavior owing to surface criticality. In the case of large grain sizes $\xi_{g}$, the corresponding critical exponent $\beta_{s}=0.8 \pm 0.04$ agrees with the surface critical exponent of the three-dimensional Ising system. With decreasing grain sizes $\xi_{g}$, however, the corresponding decrease of $\beta_{s}$ was attributed ${ }^{10}$ to either a finitesize effect, in accordance with an effective decrease in the lateral terrace size, or to an increase of the interface interaction. The latter gives rise to surface ordering which occurs
PACS number(s): 75.50.Ee, 75.70.Rf

independently of the bulk. Moreover, an increased interface interaction may explain a blocking temperature slightly above $T_{N}$.

In this paper, we investigate the temperature dependence of the exchange bias field $H_{\mathrm{EB}}$ in the $\mathrm{Fe}_{0.6} \mathrm{Zn}_{0.4} \mathrm{~F}_{2}(110) /$ $\mathrm{Fe}(14 \mathrm{~nm}) / \mathrm{Ag}(35 \mathrm{~nm})$ heterostructure by superconducting quantum interference device (SQUID, Quantum Design MPMS-5S) magnetometry. Surprisingly, we find that the blocking temperature $T_{B} \approx 63 \mathrm{~K}$ is much higher than the Néel temperature $T_{N}=46.9 \mathrm{~K}$ of the diluted bulk antiferromagnet $\mathrm{Fe}_{0.6} \mathrm{Zn}_{0.4} \mathrm{~F}_{2}$. Such a huge enhancement of $T_{B}$ with respect to $T_{N}$ cannot be explained within the framework of a small proximity effect that originates from the mutual AF/FM interaction at the interface. Instead we propose that $\mathrm{Fe}_{1-x} \mathrm{Zn}_{x} \mathrm{~F}_{2}$ clusters, with an arbitrary concentration of $\mathrm{ZnF}_{2}, 0 \leqslant x \leqslant 1$, originate from fluctuations of the diamagnetic dilution occurring during the natural growth of the $\mathrm{AF}$ bulk crystal. They appear on all length scales, where infinite clusters give rise to the well-known Griffiths phase where nonanalyticity at $T_{N} \leqslant T \leqslant T_{N}(x=0)=78.4 \mathrm{~K}$ was predicted as a rigorous theoretical result. ${ }^{11}$ However, finite clusters, which are already far more frequent than exponentially rare clusters of infinite size, are expected to provoke a local enhancement of the exchange bias. Here we present an analysis of the temperature dependence of the integral exchange-bias effect. To this end the local contributions to the AF surface magnetization, which exhibit critical behavior are averaged with respect to a phenomenological cluster distribution under the assumption of local quasicritical temperatures.

The $\mathrm{Fe}_{0.6} \mathrm{Zn}_{0.4} \mathrm{~F}_{2}(110) / \mathrm{Fe}(14 \mathrm{~nm}) / \mathrm{Ag}(35 \mathrm{~nm})$ sample is grown at $T=425 \mathrm{~K}$ under ultra high-vacuum (UHV) conditions by depositing 14-nm Fe on top of the compensated (110) surface of the diamagnetically diluted AF substrate. The metallic thin film is deposited on an $\mathrm{Fe}_{0.6} \mathrm{Zn}_{0.4} \mathrm{~F}_{2}$ single crystal which has been polished to optical flatness with $0.3-\mu \mathrm{m}$ diamond paste before transferring into the UHV chamber. Atomic force microscopy investigations of polished (001) surfaces of pure $\mathrm{FeF}_{2}$ single crystals exhibit rough surfaces of such AF insulating substrates, with a corrugation of $0.8 \mathrm{~nm}$ on a local scale up to $0.3 \mu \mathrm{m}$ while a full line scan of $10-\mu \mathrm{m}$ length contains contributions of deep and rare scratches which give rise to a corrugation of $4.8 \mathrm{~nm}$. After cooling in a field of $\mu_{0} H_{\mathrm{fc}}=5 \mathrm{mT}$ applied parallel to the 


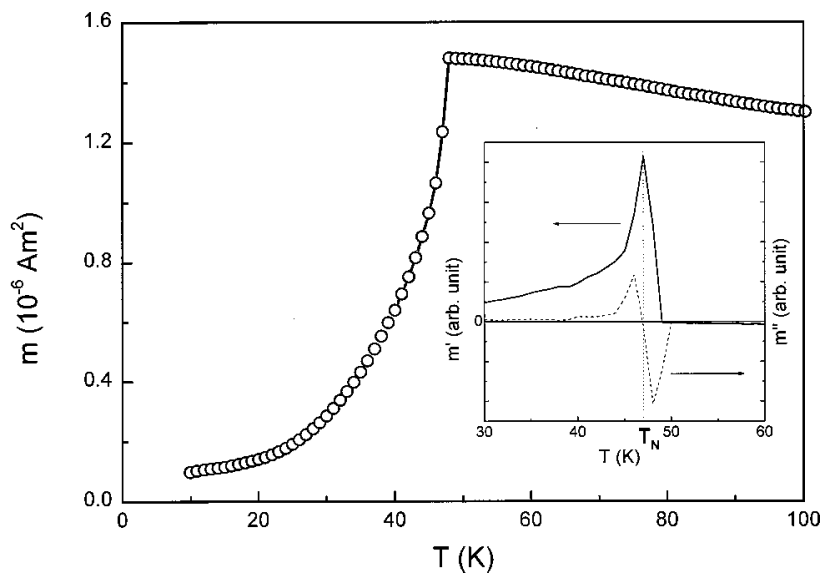

FIG. 1. Temperature dependence of the magnetic moment $m$ of $\mathrm{Fe}_{0.6} \mathrm{Zn}_{0.4} \mathrm{~F}_{2}(110) / \mathrm{Fe}(14 \mathrm{~nm}) / \mathrm{Ag}(35 \mathrm{~nm})$. The inset shows the first (solid line) and second (broken line) derivatives with respect to temperature, thus defining the Néel temperature of the $\mathrm{Fe}_{0.6} \mathrm{Zn}_{0.4} \mathrm{~F}_{2}(110)$ substrate, $T_{N}=46.9 \mathrm{~K}$.

planar [110] direction from $T=100 \mathrm{~K}$ to the respective target temperature, the exchange-bias field is determined from the shift of the hysteresis loop.

Figure 1 shows the temperature dependence of the magnetic moment $m$ of the heterostructure. The Curie temperature of $\mathrm{Fe}$ is much higher than the global $T_{N}$ of $\mathrm{Fe}_{0.6} \mathrm{Zn}_{0.4} \mathrm{~F}_{2}$. Hence the magnetic moment of Fe remains almost constant at all temperatures under investigation, and the $m$ vs $T$ dependence mainly reflects the temperature dependence of the parallel magnetic susceptibility of the antiferromagnet. However, the steep increase in the vicinity of $T_{N}$, on the one hand, and the delayed decay of the magnetic moment above $T_{N}$, on the other hand, very probably originate from the residual interface interaction. The inset of Fig. 1 exhibits the first and second derivatives of $m(T)$, which indicate the Néel temperature $T_{N}=46.9 \mathrm{~K}$ of the $\mathrm{Fe}_{0.6} \mathrm{Zn}_{0.4} \mathrm{~F}_{2}$ substrate. In contrast with this global AF critical temperature, however, the $H_{\mathrm{EB}}$ vs $T$ dependence in Fig. 2 (open circles) shows a long tail above $T_{N}$ with a blocking temperature $T_{B} \approx 63 \mathrm{~K}$.

The authors of Ref. 10 pointed out that in a $\mathrm{FeF}_{2}-\mathrm{Fe}$ system the temperature dependence of $H_{\mathrm{EB}}$ should be directly proportional to the AF surface order parameter. They implicitly made the plausible assumption that the magnetic moment $S_{\mathrm{AF}}$ possesses the $T$ dependence of the AF surface order parameter. In order to take into account the influence exerted by perturbations such as like strain and disorder on the EB effect, they introduced the "rounded" power law

$$
H_{\mathrm{EB}}(T)=H_{\mathrm{EB} 0} \int_{0}^{\infty} t^{\beta_{s}} P\left(T_{C}\right) d T_{C},
$$

where $t=1-T / T_{C}$ is the reduced temperature for $T<T_{C}$, and $t=0$ for $T>T_{C}$, while $P\left(T_{C}\right)$ is the critical temperature distribution. A narrow Gaussian distribution function

$$
P\left(T_{C}\right)=\frac{1}{\delta \sqrt{2 \pi}} \exp \left[-\left(T_{C}-T_{C 0}\right)^{2} / 2 \delta^{2}\right]
$$

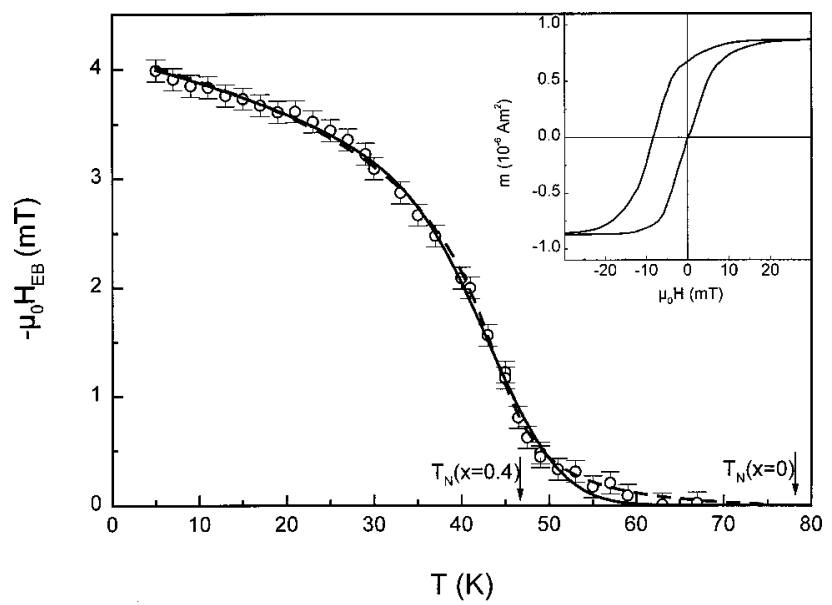

FIG. 2. Temperature dependence of the exchange bias field $\mu_{0} H_{\mathrm{EB}}$ (circles) obtained from magnetic hysteresis curves at the respective target temperatures $5 \mathrm{~K} \leqslant T \leqslant 80 \mathrm{~K}$ after cooling in a freezing field of $5 \mathrm{mT}$. The inset shows a typical hysteresis curve measured at $T=5 \mathrm{~K}$. The solid and dashed lines show the results of the best fits of Eq. (1) involving Gaussian and Lorentzian distribution functions, respectively. Arrows at 46.9 and $78.4 \mathrm{~K}$ indicate the Néel temperatures of the diluted and the pure antiferromagnet, respectively.

has been used for a best fit of the $H_{\mathrm{EB}}$ vs $T$ data, where $H_{\mathrm{EB} 0}, T_{C 0}, \beta_{s}$, and $\delta$ are the fitting parameters.

In contrast with the assumption of weak perturbations in $\mathrm{FeF}_{2}$, however, we stress the fact that in our case the diamagnetic dilution is a strong and intrinsic perturbation. It gives rise to a broad distribution function even in the case of an ideal antiferromagnet with random site dilution.

Previously, we showed that the dilution-induced Griffiths phase of the antiferromagnet $\mathrm{Fe}_{1-x} \mathrm{Zn}_{x} \mathrm{~F}_{2}$ gives rise to significant deviations from the Curie-Weiss behavior which is naively expected at $T>T_{N} \cdot{ }^{12}$ However, Griffiths-type clusters give rise to a continuous series of local phase transitions with quasicritical temperatures $T_{c}$ within $T_{N}(x) \leqslant T_{c} \leqslant T_{N}$ $(x=0)$.

In view of this experience, we fit Eq. (1) to the experimental data of Fig. 2 (circles) while alternatively using two phenomenological distribution functions, viz., the Gaussian distribution of Eq. (2) and the Lorentzian distribution

$$
P\left(T_{C}\right)=\left\{\begin{array}{c}
\frac{\varepsilon / 2}{\left[\varepsilon^{2}+\left(T_{C}-T_{C 0}\right)^{2}\right] \arctan \left\{\left[T_{N}(0)-T_{C 0}\right] / \varepsilon\right\}} \\
\quad \text { if } T_{c} \leqslant T_{N}(x=0) \\
0 \quad \text { if } T_{c}>T_{N}(x=0)
\end{array} .\right.
$$

Here $T_{N}(0)=78.4 \mathrm{~K}$ is the Néel temperature of the pure $\mathrm{FeF}_{2}$ while $H_{\mathrm{EB} 0}, T_{C 0}, \beta_{s}, \delta$, and $\varepsilon$ are the fitting parameters. The Lorentzian distribution function was used previously in order to model the $T_{c}$ distribution involved in the analogous phenomenon of a field-induced Griffiths phase. ${ }^{13}$ It is normalized under the constraint $\int_{T c 0}^{T_{N}(0)} P\left(T_{c}\right) d T_{c}=\frac{1}{2}$, where a symmetric distribution with respect to $T_{C 0}$ is assumed. $T_{C 0}$ describes the centers of gravity of the distribu- 
TABLE I. List of the parameters resulting from the best fits of Eq. (1) to the $\mu_{0} H_{\mathrm{EB}}$ vs $T$ data (Fig. 2, circles) involving Gaussian and Lorentzian distribution functions, respectively.

\begin{tabular}{ccccc}
\hline \hline $\begin{array}{c}\mu_{0} H_{E B 0} \\
(\mathrm{mT})\end{array}$ & $\begin{array}{c}T_{C 0} \\
(\mathrm{~K})\end{array}$ & $\beta_{s}$ & $\begin{array}{c}\delta \text { or } \varepsilon \\
(\mathrm{K})\end{array}$ \\
\hline
\end{tabular}

Gaussian distribution $\quad 4.1 \pm 0.1 \quad 45.0 \pm 1.5 \quad 0.22 \pm 0.04 \quad 6.1 \pm 1.6$ $\begin{array}{lllll}\text { Lorentzian distribution } & 4.1 \pm 0.1 & 44.4 \pm 1.3 & 0.19 \pm 0.03 & 4.0 \pm 0.1\end{array}$

tion functions, respectively, and is expected to be in the vicinity of the global transition temperature $T_{N}(x)=46.9 \mathrm{~K}$.

Figure 2 shows the results of the best fits of Eq. (1) involving a Lorentzian (dashed line) and a Gaussian distribution (solid line), respectively. Inspection shows that, in particular, the tail of the $H_{\mathrm{EB}}$ vs $T$ data at $T>50 \mathrm{~K}$ is modeled more accurately by the Lorentzian distribution. This is corroborated by the resulting fitting parameters which profit from smaller uncertainties in comparison with the parameters which result from the Gaussian distribution. The resulting fitting parameters are listed in Table I. Here we are going to discuss the parameters. Substitution of $t=1$ into Eq. (1) yields the physical meaning of $\mu_{0} H_{\mathrm{EB} 0}$, which is the zerotemperature limit of the exchange-bias field. Hence $\mu_{0} H_{\mathrm{EB} 0}$ $\approx 4 \mathrm{mT}$ is in accordance with the low-temperature value of the $\mu_{0} H_{\mathrm{EB} 0}$ vs $T$ data shown in Fig. 2. As expected, $T_{C 0}$ $=45$ and $44.4 \mathrm{~K}$, respectively, are close to the global transition temperature $T_{N}(x=0.4)=46.9 \mathrm{~K}$. The parameters $\delta$ $=6.1$ and $\varepsilon=4.0$ characterize the widths of the Gaussian and Lorentzian distribution functions, respectively. Physically the most interesting parameters are the surface critical exponents $\beta_{s}=0.22$ and 0.19 for the Gaussian and Lorentzian distributions, respectively. These values are surprisingly low in comparison to $\beta_{s}=0.8$, the surface critical exponent of the threedimensional Ising system found in the $\mathrm{FeF}_{2}-\mathrm{Fe}$ bilayer system. ${ }^{10}$ However, it is known that surface critical behavior depends on the ratio between the surface coupling $J_{s}$ and the bulk coupling constant $J_{b}, r=J_{s} / J_{b} \cdot{ }^{10,14,15}$ The phase diagram of the semi-infinite Ising model exhibits a critical ratio at $r_{c} \approx 1.50 .^{14,16}$ In the case $r<r_{c}$ an "ordinary transition" occurs, which depends on the bulk critical behavior. At $r$ $>r_{c}$ a surface transition occurs which is independent of the bulk transition, while a so-called "special transition" occurs at the multicritical point $r_{c}{ }^{15}$

In our case, $\beta_{s}$ is quite close to the value of the special transition where $\beta_{s}=0.237$ and 0.26 were reported in Refs. 16 and 17, respectively. As pointed out in the work of Ref. $10, \beta_{s}$ decreases with increasing interface exchange coupling because the surface spins tend to order independently of the bulk, i.e., with an exponent closer to the two-dimensional Ising value $\beta_{s}=\frac{1}{8} \cdot{ }^{18}$ In the case of the diluted antiferromagnet this tendency may be enhanced, because the diamagnetic dilution gives rise to broken magnetic "bonds." Hence the interaction of an AF surface moment with neighbors from the AF bulk is effectively reduced with respect to the exchange interaction between the AF and FM moments at the interface. Therefore the ratio of the effective surface and bulk interaction constants increases thus giving rise to a reduced $\beta_{s}$.

In conclusion, we have investigated the temperature dependence of exchange-bias field in $\mathrm{Fe}_{1-x} \mathrm{Zn}_{x} \mathrm{~F}_{2}(110) /$ $\mathrm{Fe} 14 \mathrm{~nm} / \mathrm{Ag} 35 \mathrm{~nm}, x=0.4$. The blocking temperature is located more than $16 \mathrm{~K}$ above the Néel temperature of the diluted bulk antiferromagnet. The unusual enhancement of the blocking temperature is attributed to a wide distribution of quasicritical temperatures which correspond to clusters of various diamagnetic dilution $0 \leqslant x \leqslant 1$. The clusters originate from fluctuations of the dilution which are involved in the natural growth process. Clusters of infinite size (i.e., on the length scale of the sample) give rise to the well-known Griffiths phase. However, an enhancement of the blocking temperature requires only very frequently occurring clusters of finite size. Within the framework of this cluster model, the experimental data are fitted on averaging all local components which possess surface critical behavior. The resulting critical exponent $\beta_{s} \approx 0.2$ is close to the value predicted for the so-called special transition.

Work supported by the Deutsche Forschungsgemeinschaft through SFB 491.
${ }^{1}$ J. Nogués and I. K. Schuller, J. Magn. Magn. Mater. 192, 203 (1999)

${ }^{2}$ A. E. Berkowitz and K. Takano, J. Magn. Magn. Mater. 200, 552 (1999).

${ }^{3}$ W. H. Meiklejohn and C. P. Bean, Phys. Rev. 105, 904 (1957).

${ }^{4}$ Ch. Binek, A. Hochstrat, and W. Kleemann, J. Magn. Magn. Mater. 234, 353 (2001).

${ }^{5}$ X. W. Wu and C. L. Chien, Phys. Rev. Lett. 81, 2795 (1998).

${ }^{6}$ C. Hou, H. Fujiwara, K. Zhang, A. Tanaka, and Y. Shimizu, Phys. Rev. B 63, 024411 (2000).

${ }^{7}$ R. Jungblut, R. Coehoorn, M. T. Johnson, J. van de Stegge, and R. Reinders, J. Appl. Phys. 75, 6659 (1994).

${ }^{8}$ U. Nowak, A. Misra, and K. D. Usadel, J. Appl. Phys. 89, 7269 (2001).

${ }^{9}$ P. J. van der Zaag, Y. Ijiri, J. A. Borchers, L. F. Feiner, R. M.
Wolf, J. M. Gaines, R. W. Erwin, and M. A. Verheijen, Phys. Rev. Lett. 84, 6102 (2000).

${ }^{10}$ D. Lederman, J. Nogues, and I. K. Schuller, Phys. Rev. B 56, 2332 (1997).

${ }^{11}$ R. B. Griffiths, Phys. Rev. Lett. 23, 17 (1969).

${ }^{12}$ Ch. Binek and W. Kleemann, Phys. Rev. B 51, 12888 (1995).

${ }^{13}$ Ch. Binek, S. Kuttler, and W. Kleemann, Phys. Rev. Lett. 75, 2412 (1995).

${ }^{14} \mathrm{~K}$. Binder, in Phase Transitions and Critical Phenomena, edited by C. Domb and J. L. Lebowitz (Academic, London, 1983), Vol. VIII, p. 1.

${ }^{15}$ M. Pleimling and W. Selke, Eur. Phys. J. B 1, 385 (1998).

${ }^{16}$ C. Ruge, S. Dunkelmann, and F. Wagner, Phys. Rev. Lett. 69, 2465 (1992).

${ }^{17}$ H. W. Diehl and M. Shpot, Phys. Rev. Lett. 73, 3431 (1994).

${ }^{18}$ L. Onsager, Phys. Rev. 65, 117 (1944). 